\title{
GREENSTICK FRACTURE OF THE UPPER END OF THE ULNA WITH DISLOCATION OF THE RADIO-HUMERAL JOINT OR DISPLACEMENT OF THE SUPERIOR RADIAL EPIPHYSIS
}

\author{
P. R. Wright, Margate, England
}

The purpose of this paper is to describe an injury of childhood which has received little notice in the literature.

\section{CASE REPORTS}

Case 1-A boy of four fell on to his right arm, injuring his elbow. Radiographs showed a greenstick fracture of the upper end of the ulna with extension deformity, and forward displacement of the upper end of the radius (Fig. 1). The fracture was reduced by manipulation under general anaesthesia and the arm was immobilised in plaster-of-Paris for five weeks. Two weeks after removal of the plaster he had regained a full, painless range of movement of the elbow and the forearm.



Case 1-Greenstick fracture of the ulna with extension deformity and forward displacement of the upper end of the radius.
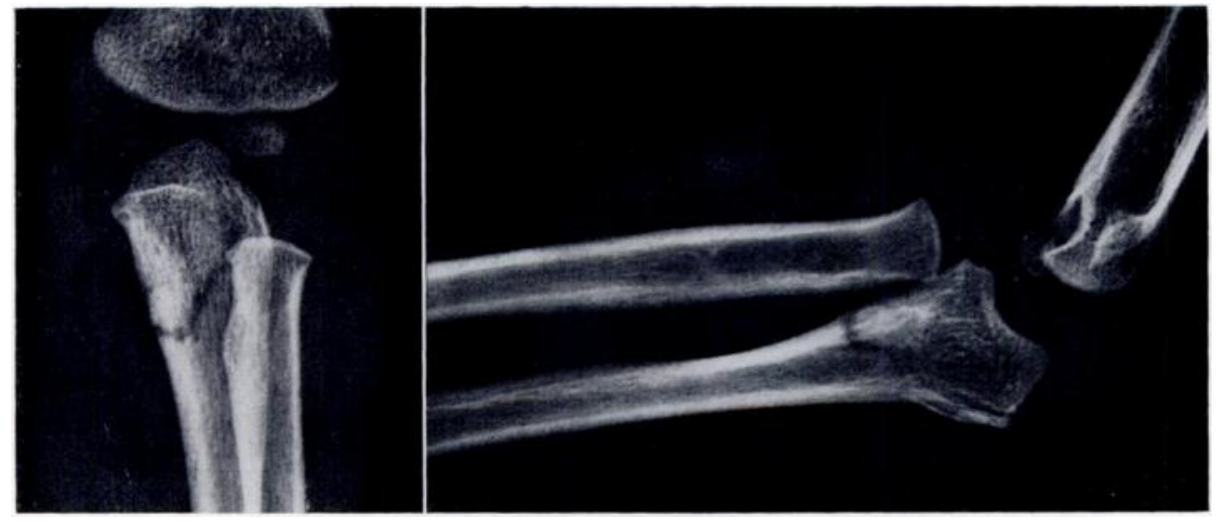

FIG. 2

Case 2-Greenstick fracture of the ulna with extension deformity and forward displacement of the upper end of the radius.

Case 2-A girl of two fell from a chair, injuring her left elbow. Radiographs showed a greenstick fracture of the upper end of the ulna with extension deformity, and forward displacement of the upper end of the radius (Fig. 2). Manipulative reduction was performed 
under general anaesthesia, and although reduction was incomplete the position was accepted. Immobilisation in plaster was maintained for three weeks. Two weeks after removal of the plaster the child had regained a full range of painless movement of the elbow and forearm.

Case 3-A boy of six injured his right arm while doing physical training at school. $\mathrm{He}$ complained of pain in the elbow, and radiographs showed a greenstick fracture of the upper end of the ulna with varus deformity and lateral displacement of the upper end of the radius (Fig. 3). The extent of this injury was not at first recognised and the arm was immobilised in plaster without manipulation. Eight days later the correct diagnosis was made and manipulative reduction was attempted but without success. Open reduction of the ulnar fracture was therefore carried out, and followed by four weeks' immobilisation. After the plaster was removed a full range of flexion, extension and supination was rapidly regained, but pronation remained slightly limited ( 70 degrees compared with 90 degrees on the other side).

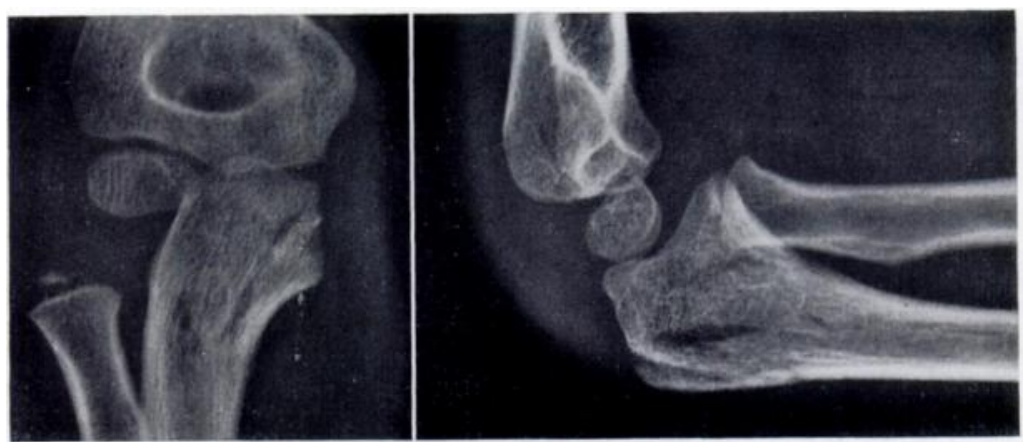

FIG. 3

Case 3-Greenstick fracture of the ulna with varus deformity and lateral displacement of the upper end of the radius.

Case 4-A girl of ten fell down a flight of steps at school and injured her right elbow. Radiographs showed a greenstick fracture of the upper end of the ulna with valgus deformity and fracture-separation of the upper radial epiphysis (Fig. 4). Open reduction of the epiphysis was carried out, together with manipulation of the ulnar fracture, and immobilisation in plaster was maintained for three weeks. On removal of the plaster it became clear that reduction of the ulnar fracture was incomplete and that there was about 15 degrees of cubitus valgus. This position was accepted. Flexion and extension of the elbow rapidly returned to normal but pronation and supination remained limited. Further radiographs four months after injury showed that the ulnar fracture involved the superior radio-ulnar joint (Fig. 5), and this explained a gradually decreasing range of pronation and supination. Eighteen months after injury anterior transposition of the ulnar nerve was performed in view of the cubitus valgus. Thirty months after injury flexion and extension remained almost full, but supination and pronation were absent, the forearm being fixed at 45 degrees of supination. Radiographs suggested that fusion of the superior radio-ulnar joint had taken place (Fig. 6).

Case 5-A boy of thirteen sustained an injury to his left elbow, the details of which were unknown. Radiographs showed a greenstick fracture of the upper end of the ulna with valgus deformity and a fracture-separation of the upper radial epiphysis. There was also separation of the epiphysis of the medial humeral epicondyle. Treatment was by manipulation under general anaesthesia followed by three weeks' immobilisation in plaster. The position on removal of the plaster is shown in Figure 7. Recovery of movement of the elbow and forearm 

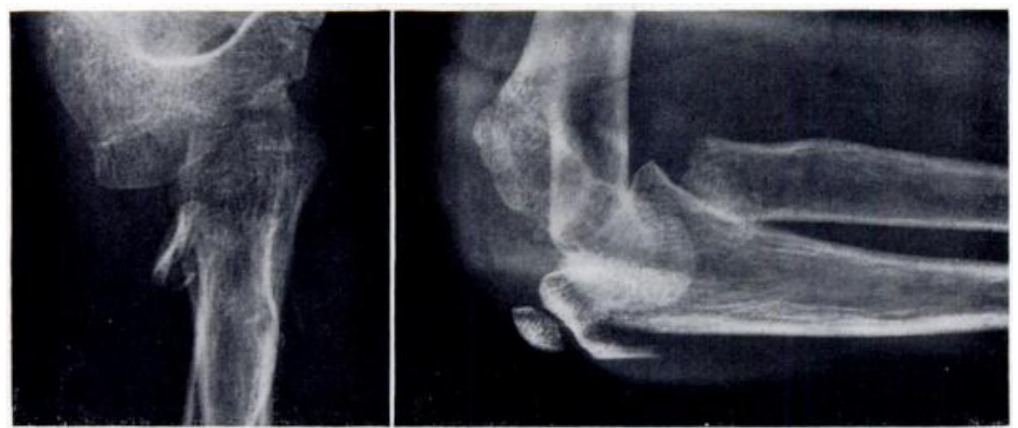

Fig. 4

Case 4-Greenstick fracture of the ulna with valgus deformity and fractureseparation of the upper radial epiphysis.
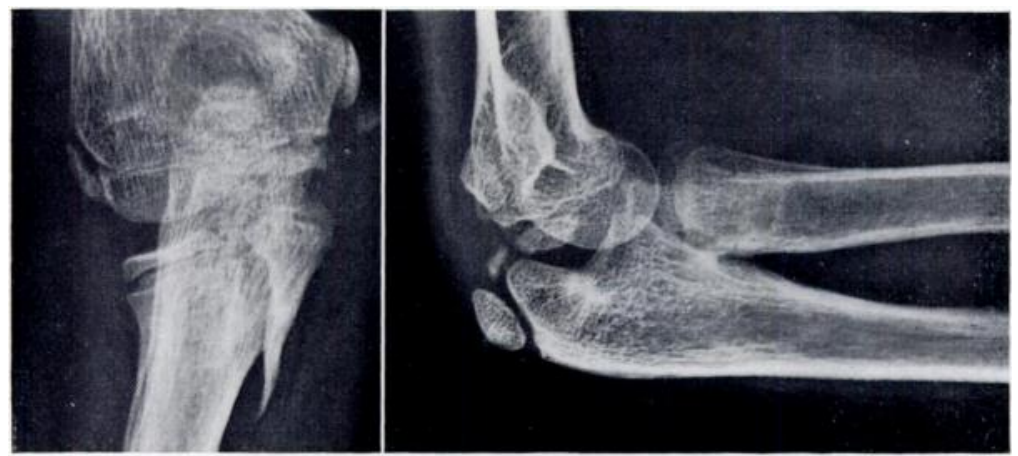

Fig. 5

Case 4-Radiographs after attempted reduction, showing incomplete reduction and involvement of the superior radio-ulnar joint by the ulnar fracture.
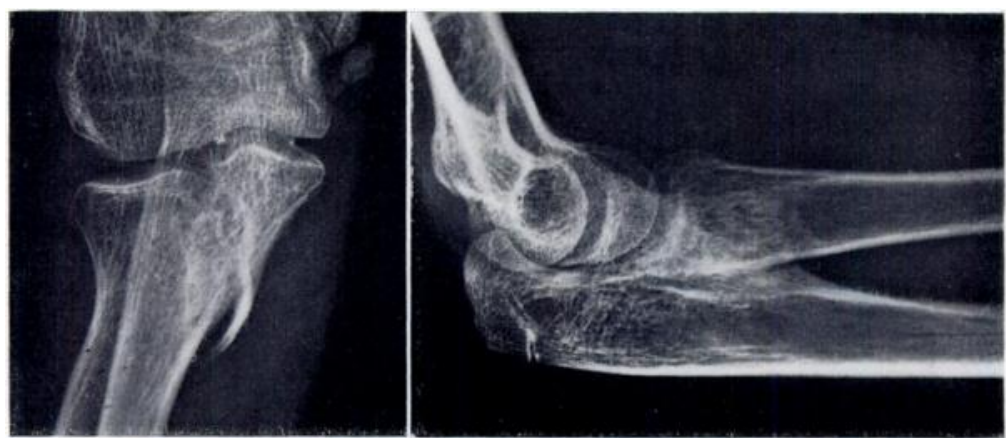

Fig. 6

Case 4-Radiographs taken thirty months after injury suggesting fusion of the superior radio-ulnar joint. 
was slow and four months after injury there was still 30 degrees loss of extension and 40 degrees loss of flexion. Pronation was limited to 20 degrees and supination to 70 degrees. At this time it became clear that traumatic ulnar neuritis was developing and anterior transposition of the ulnar nerve was performed. Further radiographs showed that the ulnar fracture had united and that involvement of the superior radio-ulnar joint had not occurred (Fig. 8).

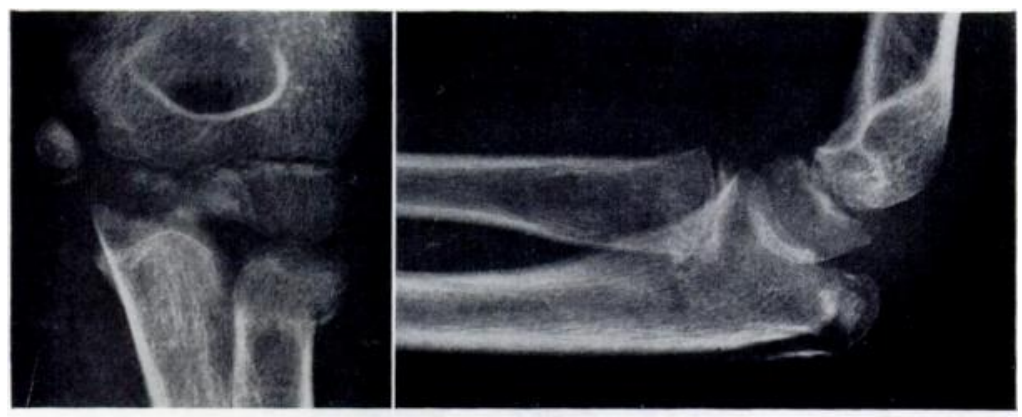

Fig. 7

Case 5-Radiographs after reduction.

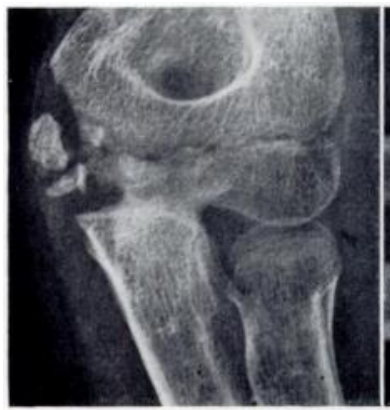

Case 5-Radiographs showing union of the ulnar fracture without involvement of the superior radio-ulnar joint.

\section{DISCUSSION}

Hume (1957) described three cases of undisplaced fracture of the olecranon in children associated with anterior dislocation of the head of the radius. The radiographs in his paper showed that in one of the cases there was a varus deformity of the ulna at the site of the fracture and the head of the radius was displaced laterally, similar to the displacement described in Case 3 above.

Beddow and Corkery (1960) described two cases of the same type, in which a greenstick fracture of the upper end of the ulna with varus deformity was accompanied by lateral dislocation of the radio-humeral joint.

The literature does not contain any other reports of this injury.

It seems clear that, no matter what the direction of angulation of the ulna may be, the ulna and the radius remain in a fixed relationship to one another while the injury is taking place. Only in this way can the correspondence between the direction of angulation of the ulna and the direction of displacement of the upper end of the radius be explained. For the same reason it seems clear that this position must be one of full supination. The most probable mechanism therefore seems to be a fall on to the outstretched hand during which the forearm is held supinated (as it might be if the child were falling backwards). The direction of angulation 
of the ulna will then be determined by the exact direction of the fall, which may produce a varus, valgus or hyperextension strain on the forearm (Fig. 9).

Hume (1957) suggested that anterior displacement of the head of the radius might be explained by a hyperextension strain on the ulna while the radius is in the position of pronation-a mechanism similar to that described by Evans (1949) in the production of the Monteggia fracture-dislocation. Such an explanation, however, could not apply to those cases with medial and lateral displacement. It seems more likely that all three types form a group, in which the position of the arm during the injury is essentially the same, but the direction of angulation is determined by the exact direction of the fall.

Complications-1) Imperfect reduction may result from failure to recognise the true nature of the injury when it is first seen. Even when it is recognised, the displacement of the ulna may not be easy to correct by manipulation (Cases 2 and 4). 2) Traumatic ulnar neuritis is a considerable danger in those cases with valgus deformity if reduction is imperfect. 3) Radial nerve injury was not seen in this series but it occurred in one of the patients with lateral displacement of the radial head described by Beddow and Corkery (1960). They considered it to be a neurapraxia resulting from traction by the displaced radius on the radial nerve as it winds through the supinator muscle. 4) Injury to the superior radio-ulnar joint must occur in all cases but complete loss of movement only occurred in one case, in which the ulnar fracture appeared to have involved the joint.



GREENSTICK FRACTURE OF ULNA WITH VARUS DEFORMITY AND LATERAL DISPLACEMENT OF RADIAL HEAD

Fig. 9

To illustrate the mechanism of this injury and the Monteggia injury.

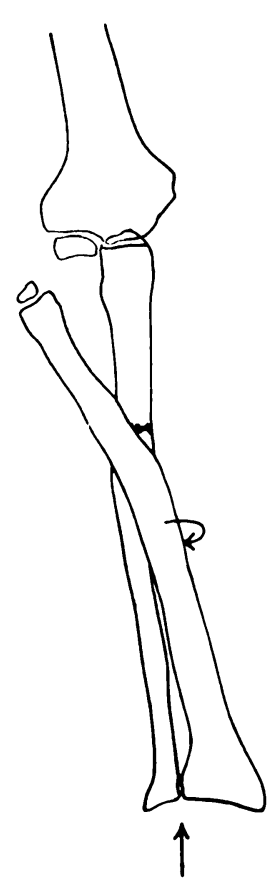

MONTEGGIA INJURY

\section{SUMMARY}

1. Five cases of greenstick fracture of the upper end of the ulna with dislocation of the radio-humeral joint are described.

2. Although the direction of angulation of the fracture and the corresponding displacement of the upper end of the radius may be lateral, medial or anterior, it is suggested that all five cases form a group in which the mechanism of injury is essentially the same. This mechanism is considered to be a fall on the outstretched hand with the forearm held in supination.

3. The complications of the injury are described.

I should like to thank Mr W. A. Watt Maney for permission to include Case 5.

\section{REFERENCES}

Beddow, F. H., and Corkery, P. H. (1960): Lateral Dislocation of the Radio-humeral Joint with Greenstick Fracture of the Upper End of the Ulna. Journal of Bone and Joint Surgery, 42-B, 782.

Evans, E. M. (1949): Pronation Injuries of the Forearm. Journal of Bone and Joint Surgery, 31-B, 578.

Hume, A. C. (1957): Anterior Dislocation of the Head of the Radius Associated with Undisplaced Fracture of the Olecranon in Children. Journal of Bone and Joint Surgery, 39-B, 508.

VOL. 45 B, No. 4, NOVEMBER 1963 